\title{
QoPS: A QoS based scheme for Parallel Job Scheduling*
}

\author{
Mohammad Islam Pavan Balaji P. Sadayappan $\quad$ D. K. Panda \\ Computer and Information Science \\ The Ohio State University \\ 2015 Neil Avenue \\ Columbus, OH 43210 \\ \{islammo, balaji, saday, panda\}@cis.ohio-state.edu
}

\begin{abstract}
Although job scheduling has been much studied, the issue of providing deadline guarantees in this context has not been addressed. In this paper, we propose a new scheme, termed as QoPS to provide Quality of Service $(Q o S)$ in the response time given to the end user in the form of guarantees in the completion time to submitted independent parallel jobs. To the best of our knowledge, this scheme is the first one to implement admission control and guarantee deadlines for admitted parallel jobs.
\end{abstract}

Keywords: QoS, Job Scheduling, Real time Deadlines, Parallel Job Scheduling

\section{Introduction}

A lot of research has focused on the problem of scheduling dynamically-arriving independent parallel jobs on a given set of resources. The metrics evaluated include system metrics such as the system utilization, throughput $[5,2]$, etc. and users metrics such as turnaround time, wait time $[9,14,3,6,13,8]$, etc. There has also been some recent work in the direction of providing differentiated service to different classes of jobs using statically or dynamically calculated priorities $[16,1]$ assigned to the jobs.

However, there has been no work addressing the provision of Quality of Service (QoS) in Parallel Job Scheduling. In the current job schedulers, the charge for a run is based on the resources used, but is unrelated to the responsiveness of the system. Thus, a 16-processor job that ran for one hour would be charged for 16 CPU-hours irrespective of whether the turn-around time were one hour or one day. Further, on most systems, even if a user is willing to pay more to get a quicker turn-around on an urgent job, there is no mechanism to facilitate that. Some systems, e.g. NERSC [1] offer

${ }^{*}$ This research is supported in part by NSF grants \#CCR-0204429 and \#EIA-9986052 different queues which have different costs and priorities: in addition to the normal priority queue, a high priority queue with double the usual charge, and a low priority queue with half the usual charge. Jobs in the high priority queue get priority over the normal queue, until some threshold on the number of serviced jobs is exceeded. Such a system offers the users some choice, but does not provide any guarantee on the response time. It would be desirable to implement a charging model for a job with two components: one based on the actual resource usage, and another based on the responsiveness sought. Thus if two users with very similar jobs submit them at the same time, where one is urgent and the other is not, the urgent job could be provided quicker response time than the non-urgent job, but would be charged more.

We view the overall issue of providing QoS for job scheduling in terms of two related aspects, which however can be decoupled:

- Cost Model for Jobs: The quicker the sought response time, the larger should be the charge. The charge will generally be a function of many factors, including the resources used and the load on the system.

- Job Scheduling with Response-time Guarantees: If jobs are charged differently depending on the response time demanded by the user, the system must provide guarantees of completion time. Although deadlinebased scheduling has been a topic of much research in the real-time research community, it has not been much addressed in the context of job scheduling.

In this paper, we address the latter issue (Job Scheduling with Response-time Guarantees) by providing Quality of Service (QoS) in the response time given to the end-user in the form of guarantees in the completion time to the submitted independent parallel applications. We do not explicitly consider the cost model for jobs; the way deadlines are associated with jobs in our simulation studies is explained in the subsequent sections. 
At this time, the following open questions arise:

- How practical is a solution to this problem?

- What are the trade-offs involved in such a scheme compared to a non-deadline based scheme?

- How does the imposition of deadlines by a few jobs affect the average response time of jobs that do not impose any deadlines?

- Meeting deadlines for some jobs might result in starvation of other non-deadline jobs. Does making the scheme starvation free by providing artificial deadlines to the non-deadline jobs affect the true deadline jobs?

We study the feasibility of such an idea by providing a framework, termed as QoPS (Standing for QoS for Parallel Job Scheduling), for providing QoS with job schedulers; we compare the trade-offs associated with it with respect to the existing non-deadline based schemes. We compare it to adaptations of two existing algorithms - the Slack-Based (SB) algorithm [16] and the Real-time (RT) algorithm [15], previously proposed in different contexts. The SB algorithm [16] was proposed as an approach to improve the utilization achieved by a back-filling job scheduler. On the other hand, the RT algorithm [15] was proposed in order to schedule non-periodic real-time jobs with hard deadlines, and was evaluated in a static scheduling scenario. As explained later, we adapted these two schemes to schedule jobs in a dynamic job scheduling context with deadlines.

The remaining part of the paper is organized as follows. In Section 2, we provide some background on deadline based job scheduling and how schemes implemented in the other domains can be modified to be incorporated in this domain. In Section 3, we discuss the design and implementation of a new scheduling scheme that allows deadline specification for jobs. The simulation approach to evaluate the schemes is discussed in Section 4. In Section 5, we present results of our simulation studies comparing the various schemes. In Section 6, we conclude the paper and present some possible future work.

\section{Background and Related Work}

Most earlier schemes proposed for scheduling independent parallel jobs dealt with either maximizing system metrics such as the system utilization, throughput, etc., or minimizing user metrics such as the turnaround time, wait time, slowdown, etc., or both. Some other schemes have also looked at prioritizing the jobs based on a number of statically or dynamically determined weights. In this section, we review some of the related previous work and propose modifications to these to suit the problem we are trying to solve.

\subsection{Review of Related Work}

In this subsection we present some previous work done in the context of scheduling parallel independent jobs. In the next subsection, we show how these schemes can be modified to allow users to specify hard realtime deadlines for their jobs.

\subsubsection{Slack-Based (SB) Algorithm}

The Slack-Based (SB) Algorithm, proposed by Feitelson et. al, is a backfilling algorithm used to improve the system throughput and the user response times. The main idea of the algorithm is to allow a slack or laxity for each job. The scheduler gives each waiting job a pre-calculated slack, which determines how long it may have to wait before running: 'important' and 'heavy' jobs will have little slack in comparison with others. When other jobs arrive, this job is allowed to be pushed behind in schedule time as long as it's execution is within the initially calculated laxity.

The calculation of the initial slack involves cost functions taking into consideration certain priorities associated with the job. This scheme supports both user selected and administrative priorities, and guarantees a bounded wait time for all jobs.

Though this algorithm has been proposed for improving the system utilization and the user response times, it can be easily modified to support hard real time deadlines by fixing the slack appropriately. We propose this modified algorithm in Section 2.2.1.

\subsubsection{Real Time (RT) Algorithm}

It has been shown that for dynamic systems with more than one processor, a polynomial-time optimal scheduling algorithm does not exist $[11,10,12]$. The Real Time (RT) Algorithm, proposed by Ramamritham et. al, is an approach to schedule uni-processor tasks with hard real time deadlines on multi-processor systems. The algorithm tries to meet the specified deadlines for the jobs by using heuristic functions. The tasks are characterized by worst case computation times, deadlines and resource requirements. Starting with an empty partial schedule, each step in the search extends the current partial schedule with one of the tasks yet to be scheduled. The heuristic functions used in the algorithm actively direct the search for a feasible schedule i.e., they help choose the task that extends the current partial schedule. Earliest Deadline First and Least Laxity First are examples of such heuristic functions.

In order to accomodate this algorithm into the domain of scheduling dynamically arriving parallel jobs, we have made two modifications to the algorithm. The first one is to allow parallel jobs to be submitted to the algorithm and the other is to allow dynamically arriving jobs. The details of the modified algorithm are provided in Section 2.2.2. 


\subsection{Modifications of Existing Schemes}

In this section we propose modifications to the SlackBased and Real-Time algorithms to support hard real time deadlines for the parallel jobs.

\subsubsection{Modified Slack Based (MSB) Algorithm}

Figure 1 shows the pseudo code for the modified slack based algorithm.

\section{Checking the admissibility of job $\mathrm{J}$ with Latest Start Time into an existing profile of size $\mathrm{N}$ :}

\author{
A. set cheapPrice to MAX_NUMBER \\ B. set cheapSchedule to existing schedule \\ C. for each time slot ts in the profile starting from \\ current time \\ a. Remove all the jobs from slot ts to the end \\ b. insert job $\mathrm{J}$ at slot ts \\ c. schedule each removed job one by one in \\ Ascending Scheduled Time (AST) order \\ d. Calculate the price of this new schedule \\ using the cost function. \\ e. if (price < cheapPrice) then \\ i. set cheapPrice $=$ price \\ ii. set cheapSchedule $=$ new Schedule \\ iii. Update the slack of all jobs \\ end if \\ end for \\ D. if (price != MAX_NUMBER ) then \\ Job is accepted \\ else \\ Job is rejected \\ end if
}

\section{Figure 1. The MSB Algorithm: Pseudo Code}

Compared to the original SB algorithm, MSB differs in the way the slack is determined for a given job. The original SB algorithm uses weighted user and political priorities to determine the slack. However, in the current scenario, we change this by setting the slack to be as: Slack = Deadline (Arrival Time + Run Time).

The rest of the algorithm follows the approach taken by the SB algorithm. The jobs currently present are arranged in an order decided by a heuristic function (such as Earliest Deadline First, or Least Laxity First). Once this order is fixed, the new job is inserted in each possible position in this arrangement. Thus, if there are $N$ jobs existing in the schedule, when the new job arrives, $N+1$ schedules are possible. A pre-decided cost function is used to evaluate the cost of each of these $N+1$ schedules and the one with the least cost is accepted. We can easily see that MSB is an $O(N)$ algorithm considering the evaluation of the cost function to be a constant cost. In practice, evaluating the cost function of the schedule depends on the number of jobs in the schedule and thus is a function of $N$. However, for the sake of comparison between the various algorithms and for ease of understanding, we approximate the evaluation of the cost function to be a constant value. It is to be noted that this approximation does not change the relative difference in the time complexity.

\subsubsection{Modified Real Time (MRT) Algorithm}

Figure 2 shows the pseudo code for the modified real time algorithm.

\section{Checking the admissibility of job $\mathrm{J}$ with deadline into an existing profile of size $\mathrm{N}$ :}

A. Remove all jobs from existing profile and add them into a Temporary List (TL).

B. Add the new job J into Temporary List(TL)

C. Sort temporary list according to the Heuristic function

D. Create an empty schedule without any job

E. for each job Ji from Temporary List a. Find whether job Ji is strongly feasible in the current partial schedule

b. if $\mathrm{Ji}$ is strongly feasible then

i. Add job Ji into partial schedule

ii. Remove job Ji from the temporary list and continue from step E else

i. Backtrack to the previous partial schedule

ii. if (no of backtracks > backtrack max) then

1. Job is rejected

2. Keep the old schedule and break else

continue step E with new partial schedule end if

end if

end for

F. if (all jobs are placed in the schedule)

a. Job $\mathrm{J}$ is accepted

b. Update the current schedule

end if

Figure 2. The MRT Algorithm: Pseudo Code

The RT algorithm assumes that the calculation of the heuristic function for scheduling a job into a given partial schedule takes constant time. However, this assumption only holds true for sequential (single processor) jobs (which was the focus of the algorithm). However, the scenario we are looking at in this paper relates to parallel jobs, where holes are possible in the partial schedule. In this scenario, such an assumption would not hold true. 
The Modified RT algorithm (MRT algorithm) uses the same technique as the RT algorithm but increases the time complexity to accomodate the parallel job scenario. When a new job arrives, all the jobs that have not yet started (including the newly arrived job) are sorted using some heuristic function (this function could be Earliest Deadline First, Least Laxity First, etc). Each of these jobs is inserted into the schedule in the sorted order. A partial schedule at any point during this algorithm is said to be feasible if every job in it meets its deadline. A partial schedule is said to be strongly feasible if the following two conditions are met:

- The partial schedule is Feasible

- The partial schedule would remain feasible when extended by any one of the unscheduled jobs

When the algorithm reaches a point where the partial schedule obtained is not feasible, it backtracks to a previous strongly feasible partial schedule and tries to take a different path. A certain number of backtracks are allowed, after which the scheduler rejects the job.

\section{The QoPS Scheduler}

In this section we present the QoPS Scheduler to deal with parallel job scheduling in hard real time deadline-based systems. As mentioned earlier, it has been shown that for dynamic systems with more that one processor, a polynomialtime optimal scheduling algorithm does not exist. The QoPS scheduling algorithm uses a heuristic approach to try to find feasible schedules for the jobs.

The scheduler ideally considers a system where each job arrives with a corresponding completion time deadline requirement. When each job arrives, it attempts to find a feasible schedule for the newly arrived job. A schedule is said to be feasible if it does not violate the deadline constraint for any job in the schedule, including the newly arrived job. However, it does allow a flexibility of reordering the jobs in any order as long as the resultant schedule remains feasible. Figure 3 presents the pseudo code for the QoPS scheduling algorithm.

The main difference between the MSB and the QoPS algorithm is the flexibility the QoPS algorithm offers in reordering the jobs that have already been scheduled (but not yet started).

For example, suppose jobs $\mathrm{J}_{1}, \mathrm{~J}_{2}, \ldots, \mathrm{J}_{N}$ are the jobs which are currently in the schedule but not yet started. The MSB algorithm specifies an order for the jobs as calculated by some heuristic function (the heuristic function could be least laxity first, earliest deadline first, etc). This ordering of jobs specifies the order in which the jobs have to be considered for scheduling. For the rest of the algorithm, this ordering is fixed. When a new job $\mathbf{J}_{N+1}$ arrives, the MSB algorithm tries to fit this new job in the given schedule without any change to the initial ordering of the jobs.
On the other hand, the QoPS scheduler allows flexibility in the order in which jobs are considered for scheduling. The amount of flexibility offered is determined by the K-factor denoted in the pseudo code illustrated by Figure 3 .

\section{Checking the admissibility of job $\mathrm{J}$ into an existing profile of size N:}



\section{Figure 3. The QoPS Scheduler: Pseudo Code}

When a new job arrives, it is given $\log _{2}(N)$ points in time where its insertion into the schedule is attempted, corresponding to the reserved start-times of jobs $\{0, N / 2,3 N / 4$, ... \} respectively, where $N$ is the number of jobs currently in the schedule. The interpretation of these options is as follows: For option 1 (coresponding to job 0), we start by removing all the jobs from the schedule and placing them in a temporary ordering (TL). We then sort TL according to some heuristic function (again, the heuristic function could be least laxity first, earliest deadline first, etc). Finally, we try to place the jobs in the order specified by the temporary ordering TL. For option 2, we do not start with an empty schedule. Instead, we only remove the latter $\mathrm{N} / 2$ jobs in the original schedule, chosen in scheduled start time order, place them in the temporary list TL, and sort this temporary list (based on the heuristic function). We then create a reservation for the newly arrived job, and finally generate reservations for the remaining $\mathrm{N} / 2$ jobs in the order specified by 
TL. Thus, there would be $\log N$ options of placement.

For each option given to the newly arrived job, the algorithm tries to schedule the jobs based on this temporary ordering. If a job misses its deadline, this job is considered as a critical job and is pushed to the head of the list (thus altering the temporary schedule). This altering of the temporary schedule is allowed at most ' $\mathrm{K}$ ' times; after this the scheduler decides that the new job cannot be scheduled while maintaining the deadline for all of the already accepted jobs and rejects it. This results in a time complexity of $O(K \log$ $N)$ for the QoPS scheduling algorithm.

\section{Evaluation Approach}

In this section we present the approach we took to evaluate our scheme with the other schemes and the non-deadline based EASY scheme.

\subsection{Trace Generation}

Job scheduling strategies are usually evaluated using real workload traces, such as those available at the Parallel Workload Archive [4]. However real job traces from supercomputer centers have no deadline information.

A possible approach to evaluating the QoPS scheduling strategy might be based on the methodology that was used in [15] to evaluate their real-time scheduling scheme. There randomized synthetic job sets was created in such a way that a job set could be packed into a fully filled schedule, say from time $=0$ to time $=\mathrm{T}$, with no holes at all in the entire schedule. Each job was then given an arrival time of zero, and a completion deadline of $(1+r) * T$. The value of ' $r$ ' represented a degree of difficulty in meeting the deadlines. A larger value of ' $r$ ' made the deadlines more lax. The initial synthetic packed schedule is clearly a valid schedule for all non-negative values of ' $r$ '. The real-time scheduling algorithm was evaluated for different values of ' $r$ ', over a large number of such synthesized task sets. The primary metric was the fraction of cases that a valid schedule for all tasks was found by the scheduling algorithm. It was found that as ' $r$ ' was increased, a valid schedule was found for a larger fraction of experiments, asymptotically tending to $100 \%$ as ' $r$ ' increased.

We first attempted to extend this approach to the dynamic context. We used a synthetic packed schedule of jobs, but unlike the static context evaluated in [15], we set each job's arrival time to be its scheduled start time in the synthetic packed schedule, and set its deadline beyond its start-time by $(1+r)$ times its runtime. When we evaluated different scheduling algorithms, we found that when ' $r$ ' was zero, all schemes had a $100 \%$ success rate, while the success rate dropped as ' $r$ ' was increased! This was initially puzzling, but the reason was quickly apparent - with $\mathrm{r}=0$, as each job arrives, the only possible valid placement of the new job corresponds to that in the synthetic packed schedule, and any deadline-based scheduling algorithm exactly tracks the optimal schedule. When ' $r$ ' is increased, other choices are feasible, and the schedules begin diverging from the optimal schedule, and the failure rate increases. Thus, this approach to generating the test workload is attractive in that it has a known valid schedule that meets the deadlines of all jobs; but it leads to the unnatural trend of decreasing scheduling success rate as the deadlines of jobs are made more relaxed.

Due to the above problem with the evaluation methodology used in [15], we pursue a different trace-driven approach to evaluation. We use a trace from Feitelson's archive (a 5000-job subset of the CTC trace) and first use EASY back-fill to generate a valid schedule for the jobs. Deadlines are then assigned to all jobs, based on their completion time on the schedule generated by EASY back-fill. A deadline stringency factor determines how much tight the deadline is to be set, compared to the EASY back-fill schedule. With a stringency factor of 0 , the deadlines are set to be the completion times of the jobs with the EASY back-fill schedule. With a stringency factor of 's', the deadline of each job is set ahead of its arrival time by max(runtime, (1s)*EASY-Schedule-Response-time). The metric used is the number of jobs successfully scheduled. As 's' is increased, the deadlines become more stringent. So we would expect the number of successfully scheduled jobs to decrease.

\subsection{Evaluation Content}

With the first set of simulation experiments, the three schemes (MRT, MSB and QoPS) are compared under different offered load. The load is varied by introducing a number of duplicate jobs with randomly generated arrival times. A load factor " $l$ " is varied from 1.0 to 1.6 . With $l=1.0$, only the original jobs in the CTC trace subset are used. With $1=1.2,20 \%$ of the original jobs are picked, and duplicates are introduced into the trace at random points in time. The modified trace is first scheduled using EASY back-fill, and then the deadlines for jobs are set as described above, based on the stringency factor.

After evaluating the schemes under the scenario described above, we have carried out another set of experiments under a model where only a fraction of the jobs have deadlines associated with them. This might be a more realistic practical scenario at supercomputer centers - while some of the jobs may be urgent and impose deadlines, there would likely also be other jobs that are non-urgent, with the users not requiring any deadlines. In order to evaluate the MSB, MRT, and QoPS schemes under this scenario of mixed jobs, some with user-imposed deadlines and others without, we artificially create very lax deadlines for the non-deadline jobs. While the three schemes could be run with an "infinite" deadline for the non-deadline jobs, we do not do that in order to avoid starvation of any jobs. The artificial deadline of each nondeadline job was set to $\max \left(24\right.$ hours, $\mathrm{R}^{*}$ runtime), where ' $R$ ' is a "relaxation" factor. Thus, short non-deadline jobs 
were given an artificial deadline of one day, while long jobs were given a deadline of $\mathrm{R}^{*}$ runtime. We present results for experiments with values of 2,5 , and 10 for ' $R$ '.

\section{Experimental Results}

As discussed in the previous section, the deadline-based scheduling schemes are evaluated through simulation using traces derived from the CTC trace archived at the Parallel Workloads Archive [4]. Deadlines are associated with each job in the trace as described earlier. Different offered loads were simulated by addition of a controlled number of duplicate jobs. The introduction of duplicate jobs is done incrementally, i.e. the workload for a load of 1.6 would include all the jobs in the trace for load 1.4, with the same arrival times for the common jobs. For a given load, different experiments are carried out for different values of the stringency factor ' $S$ ', with jobs having more stringent deadlines for a higher stringency factor.

\subsection{All Jobs with Deadlines}

We first present results for the scenario where all the submitted jobs have deadlines associated with them, determined as described in the previous section. The metrics measured are the total number of unadmitted jobs and the total number of lost processor-seconds from the unadmitted jobs.

Figure 4 shows the number of unadmitted jobs and lost processor-seconds for the MRT, MSB and QoPS schedules, for a stringency factor of 0.2 , as the load factor is varied from 1.0 to 1.6. It can be seen that the QoPS scheme performs better, especially at high load factors.

In the case of QoPS, as the load is increased from 1.4 to 1.6, the total number of unaccepted jobs actually decreases, even though the total number of jobs in the trace increases from 7000 to 8000 . The reason for this counter-intuitive result is as follows. As the load increases, the average wait time for jobs under EASY backfill increases nonlinearly as we approach system saturation. Since the deadline associated with a job is based on its schedule with EASY backfill, the same job will have a higher response time and hence looser deadline in a higher-load trace than in a trace with lower load. So it is possible for more jobs to be admitted with a higher-load trace than with a lower-load trace, if there is sufficient increase in the deadline. A similar and more pronounced downward trend with increasing load is observed for the unadmitted processor-seconds. This is due to the greater relative increase in response time of "heavier" jobs (i.e. those with higher processor-seconds) than lighter jobs. As the load increases, more heavy jobs are admitted and more light jobs are unable to be admitted.

The same overall trend also holds for a higher stringency factor (0.5), as seen in Figure 5. However, the performance of QoPS is closer to the other two schemes. In general, we find that as the stringency factor increases, the performance of the different strategies tends to converge. This suggests that the additional flexibility that QoPS tries to exploit in rearranging schedules is most beneficial when the jobs have sufficient laxity with respect to their deadlines.

We next look at the achieved utilization of the system, as the load is varied. As a reference, we compare the utilization for the deadline-based scheduling schemes with nondeadline job scheduling (EASY back-filling) using the same trace. Since a fraction of submitted jobs are unable to be admitted in the deadline-based schedule, clearly we can expect the achieved system utilization to be worse than the non-deadline case. Figure 6 shows the system utilization achieved for stringency factors of 0.2 and 0.5 . There is a loss of utilization of about $10 \%$ for QoPS when compared to EASY, when the stringency factor is 0.2 . With a stringency factor of 0.5 , fewer jobs are admitted, and the utilization achieved with the deadlin-based scheduling schemes drops by $5-10 \%$. Among the deadline-based schemes, QoPS and MSB perform comparably, with MRT achieving 3-5\% lower utilization at high load (load factor of 1.6).

Figure 7 shows the variation of the admittance capabilities of the three schemes for the SDSC (San Diego Super Computer Center) trace. It can be noted that the general trend of the relative performances of the schemes does not change significantly. Similarly, figure 8 shows the variation of the admittance capabilities of the three schemes using job expansion instead of job duplication as the means to increase the load. Again, it can be seen that the trend remains unchanged. These results show that the scheme is robust with respect to the workload characteristics of the trace and the load increasing mechanism. In the rest of the paper, we present only the results for the CTC trace using job duplication as the load increasing mechanism. For further results, we refer the reader to [7].

\subsection{Mixed Job Scenario}

We next consider the case when only a subset of submitted jobs have user-specified deadlines. As discussed in Section 4 , non-deadline jobs are associated with an artificial deadline that provides considerable slack, but prevents starvation. We evaluate the following combinations through simulation: a) $80 \%$ non-deadline jobs and $20 \%$ deadline jobs, and b) $20 \%$ non-deadline jobs and $80 \%$ deadline jobs. For each combination, we consider stringency factors of 0.20 and 0.50 .

Figure 9 shows the variation of the admittance of deadline jobs with offered load for the schemes, when $80 \%$ of the jobs are deadline jobs, and stringency factor is 0.2 . It can be seen that the QoPS scheme provides consistently superior performance compared to the MSB and MRT schemes, especially at high load. As with the case when all jobs were deadline jobs, when the stringency factor is increased, the performance of the different schemes tend to get more sim- 

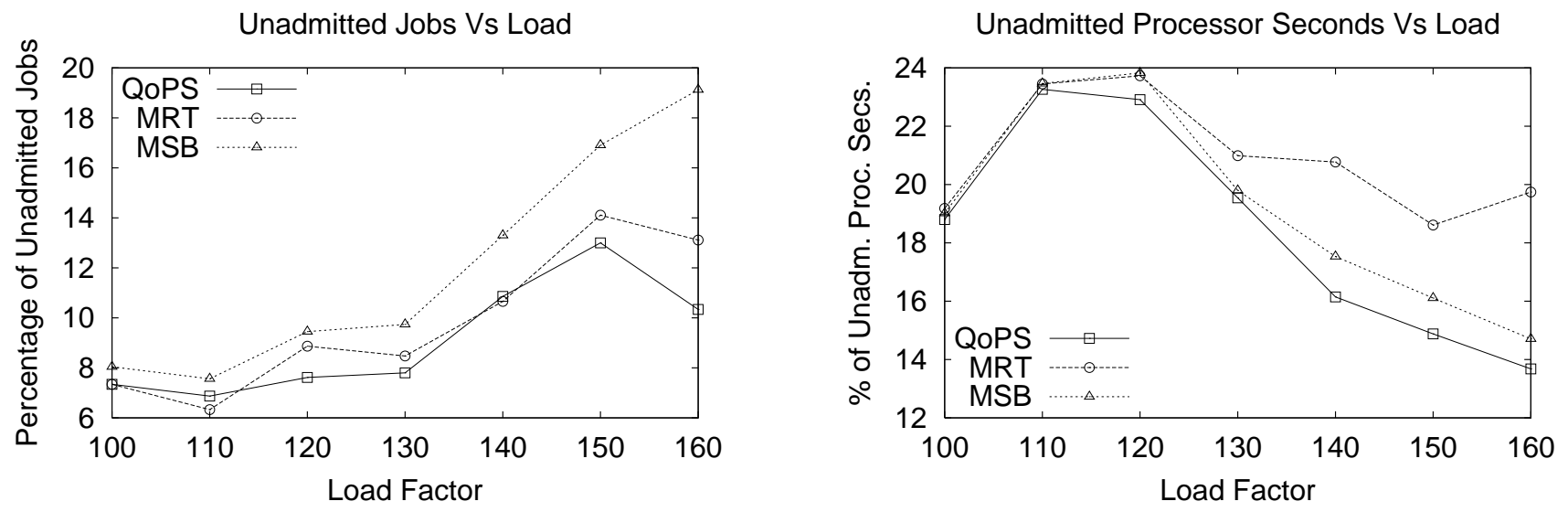

Figure 4. Admittance capacity for less stringent (Stringency Factor $=0.2$ ) deadlines (CTC trace with job duplication): (a) Unadmitted jobs, (b) Unadmitted Processor Seconds
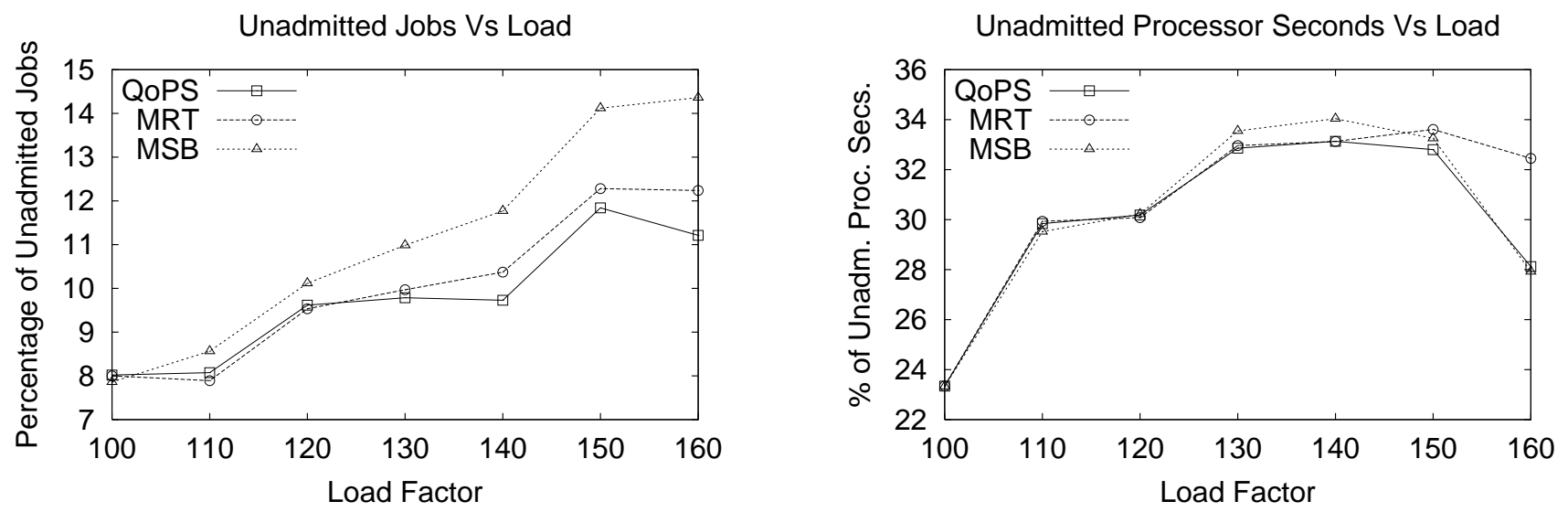

Figure 5. Admittance capacity for more stringent (Stringency Factor $=0.5$ ) deadlines (CTC trace with job duplication): (a) Unadmitted jobs, (b) Unadmitted Processor Seconds
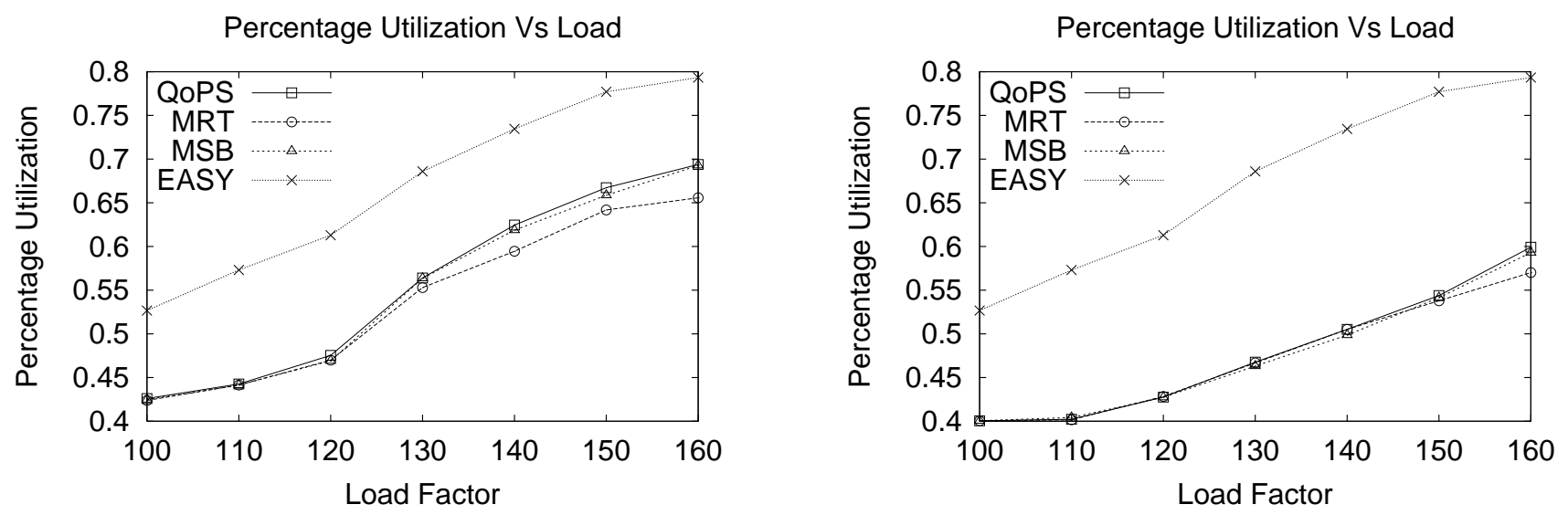

Figure 6. Utilization comparison (CTC trace with job duplication): (a) Stringency Factor $=0.2$, (b) Stringency Factor $=0.5$ 

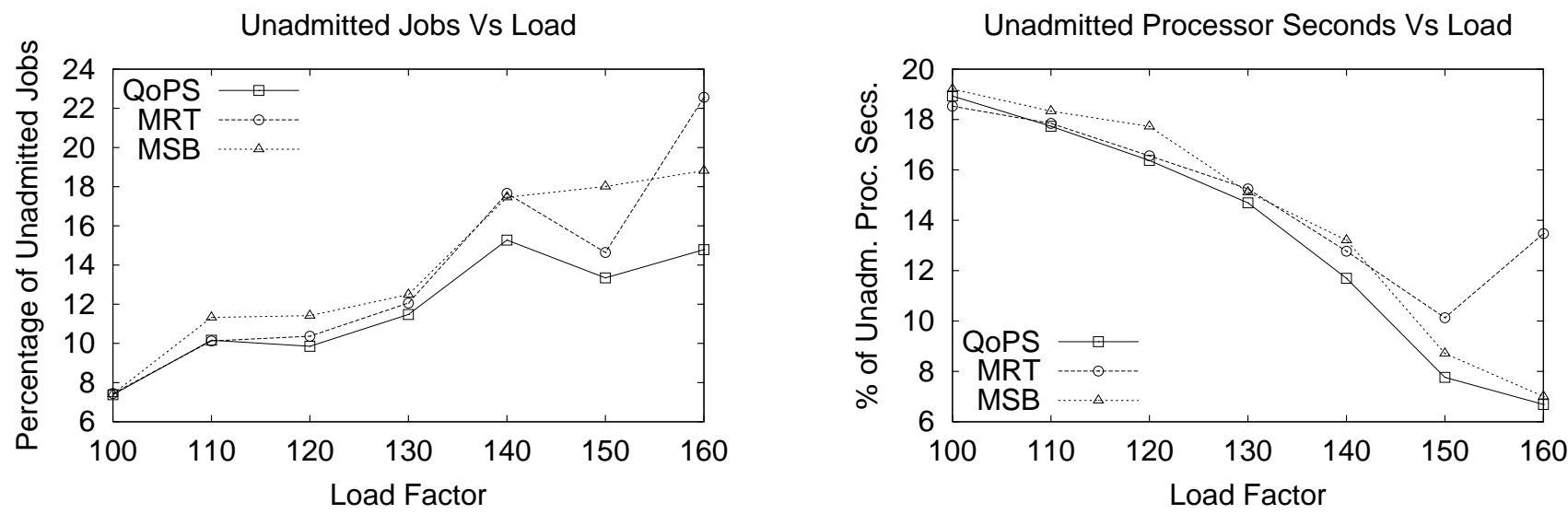

Figure 7. Admittance capacity for less stringent (Stringency Factor $=0.2$ ) deadlines (SDSC trace with job duplication): (a) Unadmitted jobs, (b) Unadmitted Processor Seconds
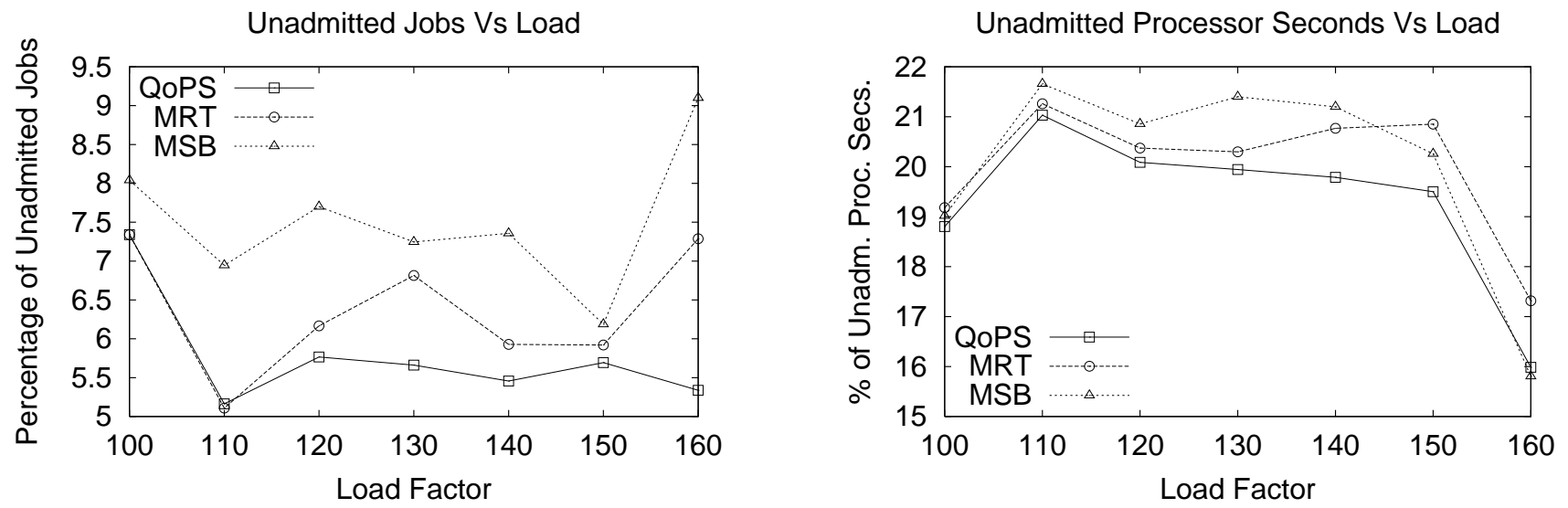

Figure 8. Admittance capacity for less stringent (Stringency Factor $=0.2$ ) deadlines (CTC trace with job expansion): (a) Unadmitted jobs, (b) Unadmitted Processor Seconds
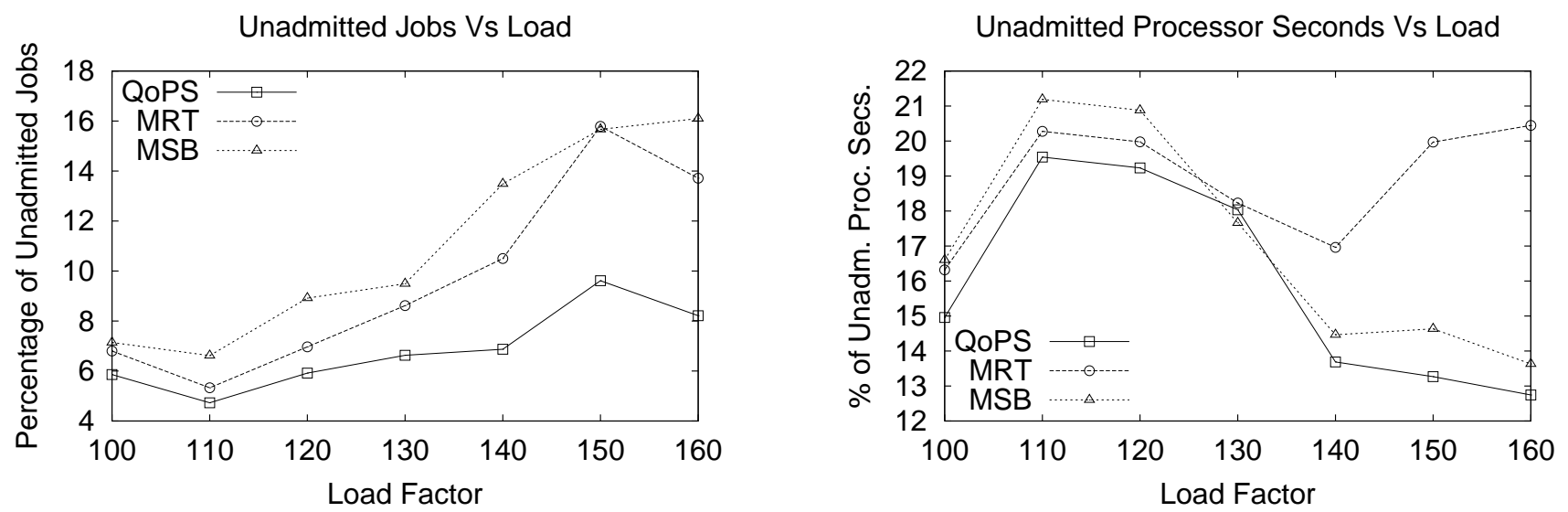

Figure 9. Admittance capacity with a mix of deadline and non-deadline jobs (Percentage of Deadline Jobs $=\mathbf{8 0} \%$ ) for less stringent (Stringency Factor $=0.2$ ) deadlines (CTC trace with job duplication):

(a) Unadmitted jobs, (b) Unadmitted Processor Seconds 

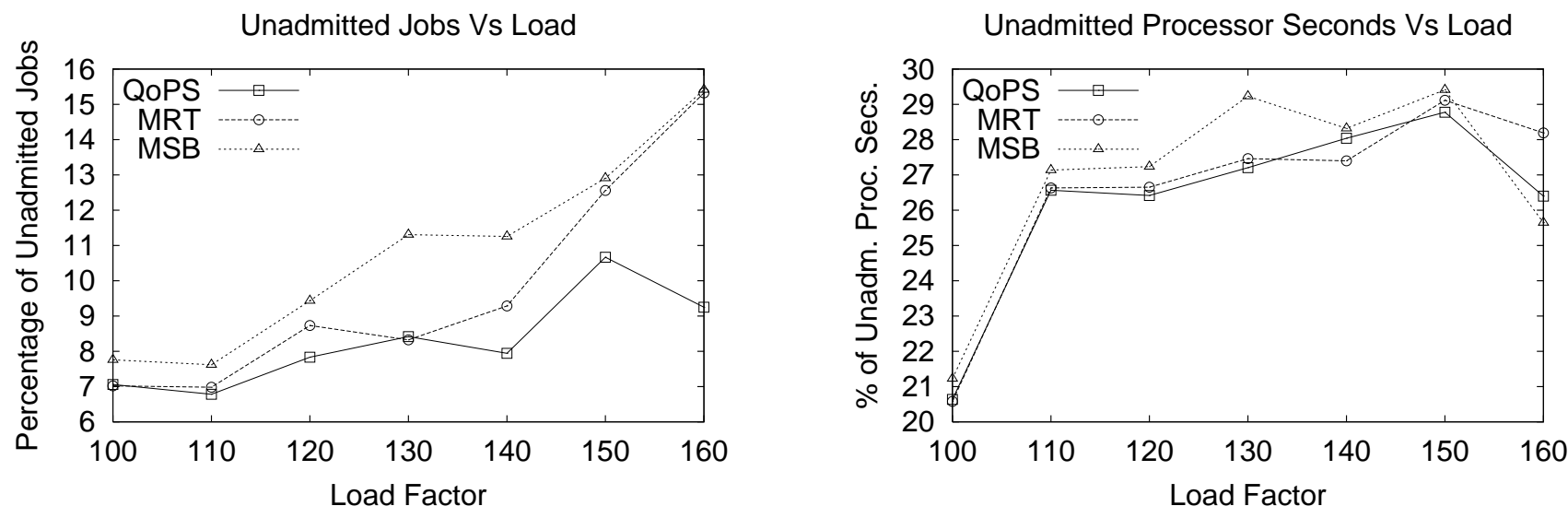

Figure 10. Admittance capacity with a mix of deadline and non-deadline jobs (Percentage of Deadline Jobs $=\mathbf{8 0} \%$ ) for more stringent (Stringency Factor $=0.5$ ) deadlines (CTC trace with job duplication): (a) Unadmitted jobs unadmitted, (b) Unadmitted Processor Seconds


Figure 11. Admittance capacity with a mix of deadline and non-deadline jobs (Percentage of Deadline Jobs $=20 \%$ ) for less stringent (Stringency Factor $=0.2$ ) deadlines (CTC trace with job duplication): (a) Unadmitted jobs, (b) Unadmitted Processor Seconds 
ilar, as shown in Figure 10.

Figures 11 and 12 present data for cases with $20 \%$ of jobs having user-specified deadlines, and stringency factors of 0.2 and 0.5 respectively. Compared to the cases with $80 \%$ of jobs being deadline-jobs, the QoPS scheme significantly outpeforms the MSB and MRT schemes, even when the stringency factor is high (0.5). This again suggests that in scenarios where many jobs have significant flexibility (here the non-deadline jobs comprise $80 \%$ of jobs and they have significant flexibility in scheduling), the QoPS scheme makes effective use of the available flexibility.

Figure 13 shows the variation of the average response time and average slowdown of non-deadline jobs with load, for the case with $80 \%$ of jobs being deadline jobs and a stringency factor of 0.2. In addition to the data for the three deadline-based scheduling schemes, data for EASY backfill is also shown. The average response time and slowdown can be seen to be lower for QoPS, MRT and MSB, when compared to EASY. This is because the delivered load for EASY is equal to the offered load (the $\mathrm{X}$-axis), whereas the delivered load for the deadline-based scheduling schemes is lower than offered load. In other words, with EASY, all the jobs are admitted, whereas with the other deadline based schemes, not all deadline jobs are admitted. This also explains the reason why the performance of QoPS appears inferior to MSB and MRT - as seen from Figure 9, the rejected load from the deadline jobs is much higher for MRT than QoPS.

When the data for the case of $20 \%$ deadline jobs is considered (Figure 14), it can be seen that the performance of QoPS has improved relative to MSB; the turnaround time is comparable or better except for a load of 1.6, and the average slowdown is lower at all loads. These user metrics are better for QoPS than MSB/MRT despite accepting a higher load (Figure 11). Data for stringency factor of 0.5 is omitted for space reasons and may be found in [7].

The achieved utilization for the different schemes as a function of load is shown in Figure 15, for a stringency factor of 0.2 . It can be seen that the achieved utilization with QoPS is roughly comparable with MSB and better than MRT, but worse than EASY. Compared to the case when all jobs had user-specified deadlines (Figure 6), the loss of utilization compared to EASY is much less - about $8 \%$ when $80 \%$ of the jobs are deadline jobs, and $2-3 \%$ when $20 \%$ of the jobs are deadline jobs.

As discussed above, a direct comparison of turnaround time and slowdown as a function of offered load is complicated by the fact that different scheduling schemes accept different numbers of jobs. A better way of comparing the schemes directly is by plotting average response time or slowdown against achieved utilization on the X-axis (instead of offered load). This is shown in Figure 16, for the case of $20 \%$ deadline jobs and stringencyfactor of 0.2 (the data for other cases may be found in [7]). It can be seen that QoPS is consistently superior to MSB and MRT. Further,
QoPS has better performance than EASY too, especially for the slowdown metric. Thus, despite the constraints of the deadline-jobs, QoPS is able to achieve better slowdown and response time for the non-deadline jobs when compared to EASY, i.e. instead of adversely affecting the non-deadline jobs, for the same delivered load, QoPS provides better performance for them, when compared to standard EASY back-fill.

\subsection{Variation of Relaxation Factor}

The last set of results we present deal with the effects of varying the "relaxation factor", R. Figure 17 shows the variation of the average response time and the average slowdown experienced by the non-deadline jobs for different relaxation factor values with $80 \%$ deadline jobs and a stringency factor of 0.2. For the non-deadline jobs, as the relaxation factor increases, the artificial deadline given to the short jobs does not change and stays at one day. However, an increase in the relaxation factor increases the slack given to the longer jobs, enabling the shorter jobs to use up this additional slack. So, it can be expected that the shorter jobs would benefit with increasing value of ' $R$ ', while the longer jobs would suffer. Following the same, the average response time of the schedule, which is weighed by the longer jobs increases with increasing ' $R$ '. On the other hand, the average slowdown of the schedule, which is weighed by the shorter jobs decreases with increasing ' $R$ '. Data for $20 \%$ deadline jobs and for a stringency factor of 0.5 can be found at [7].

\section{Conclusions and Future Work}

Scheduling dynamically-arriving independent parallel jobs on a given set of resources is a long studied problem; solutions ranging from evaluation of system and user metrics such as utilization, throughput, turnaround time, etc. to soft time-guarantees for the response time of jobs using priority based scheduling. However, a solution to the problem of providing Quality of Service (QoS) for Parallel Job Scheduling has been long overdue. In this paper, we proposed a new scheme termed as the QoPS Scheduling Algorithm to provide QoS in the response time given to the end user in the form of guarantees in the completion time to the submitted independent parallel jobs.

The current scheme does not explicitly deal with costmetrics for charging the jobs depending on the deadlines and resource usage. Also, when a job arrives, it has a number of options for placement in the schedule. The current scheme looks at each of these options in a FCFS order and does not do any kind of evaluation to see if one option is better (for the system and user metrics, such as utilization for example) than the others. We plan to extend this to define cost functions for both charging the jobs and for evaluating the different options and using the best available option. 

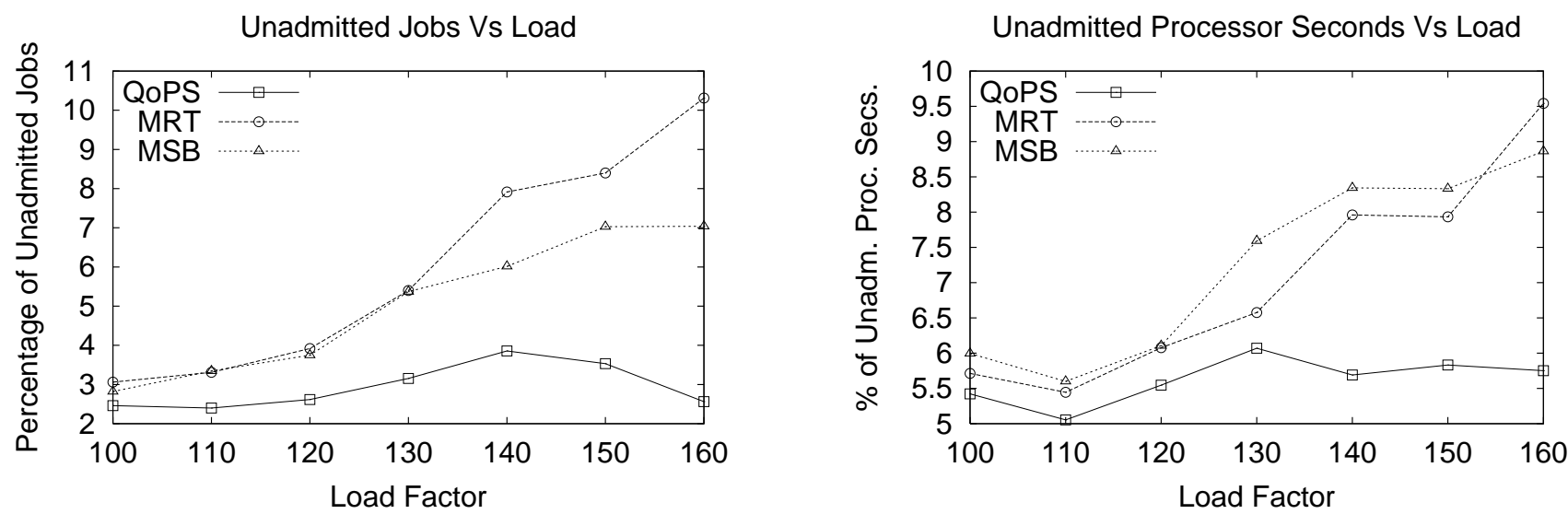

Figure 12. Admittance capacity with a mix of deadline and non-deadline jobs (Percentage of Deadline Jobs $=20 \%$ ) for more stringent (Stringency Factor $=0.5$ ) deadlines (CTC trace with job duplication): (a) Unadmitted jobs, (b) Unadmitted Processor Seconds
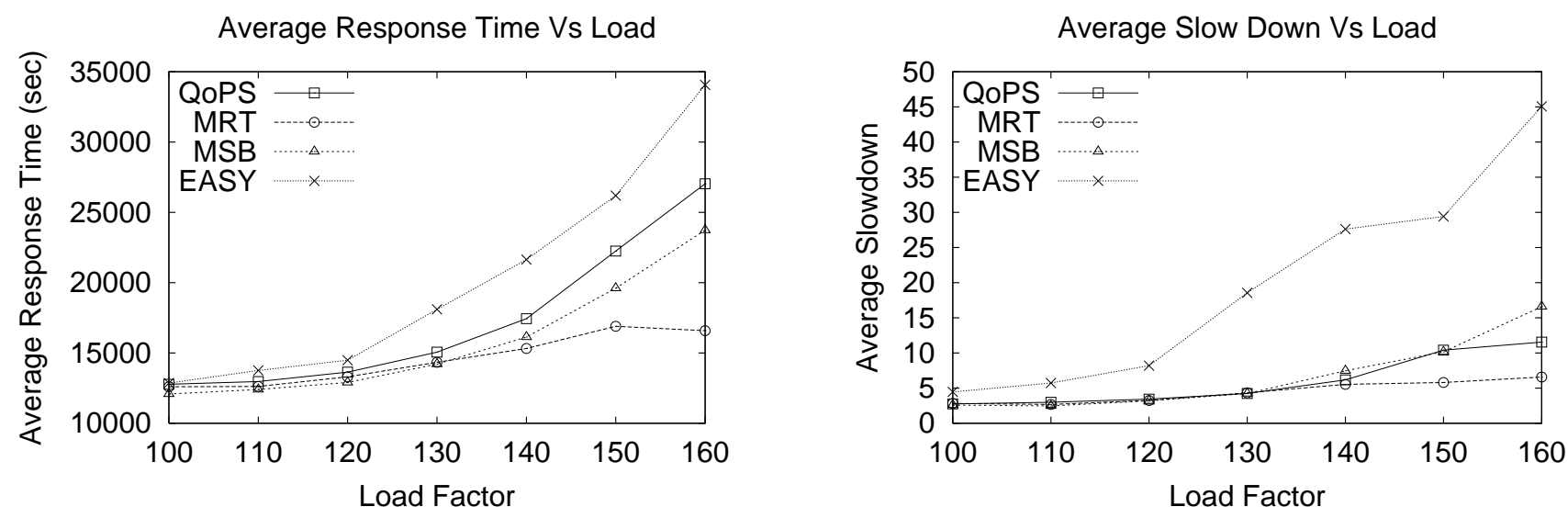

Figure 13. Performance of Non-deadline jobs with Percentage of Deadline Jobs $=80 \%$; Stringency Factor $=0.2$; CTC trace with job duplication (a) Variation of response time with Load, (b) Variation of the average slowdown with Load
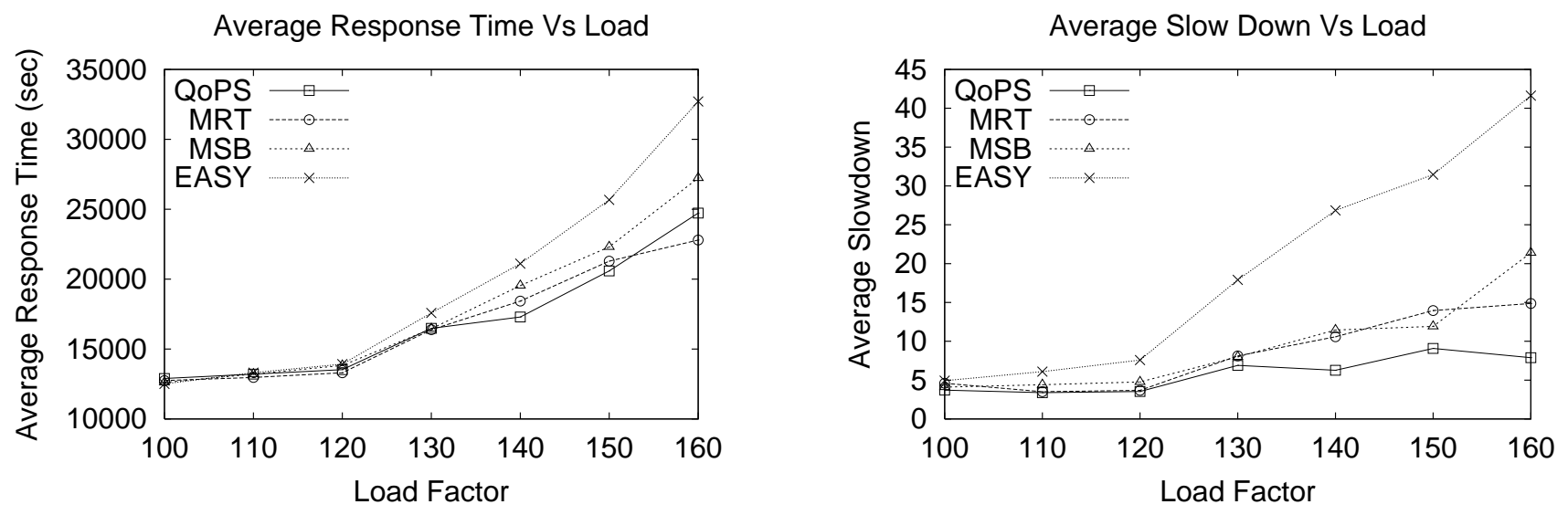

Figure 14. Performance of Non-deadline jobs with Percentage of Deadline Jobs $=20 \%$; Stringency Factor $=0.2$; CTC trace with job duplication (a) Variation of response time with Load, (b) Variation of the average slowdown with Load 

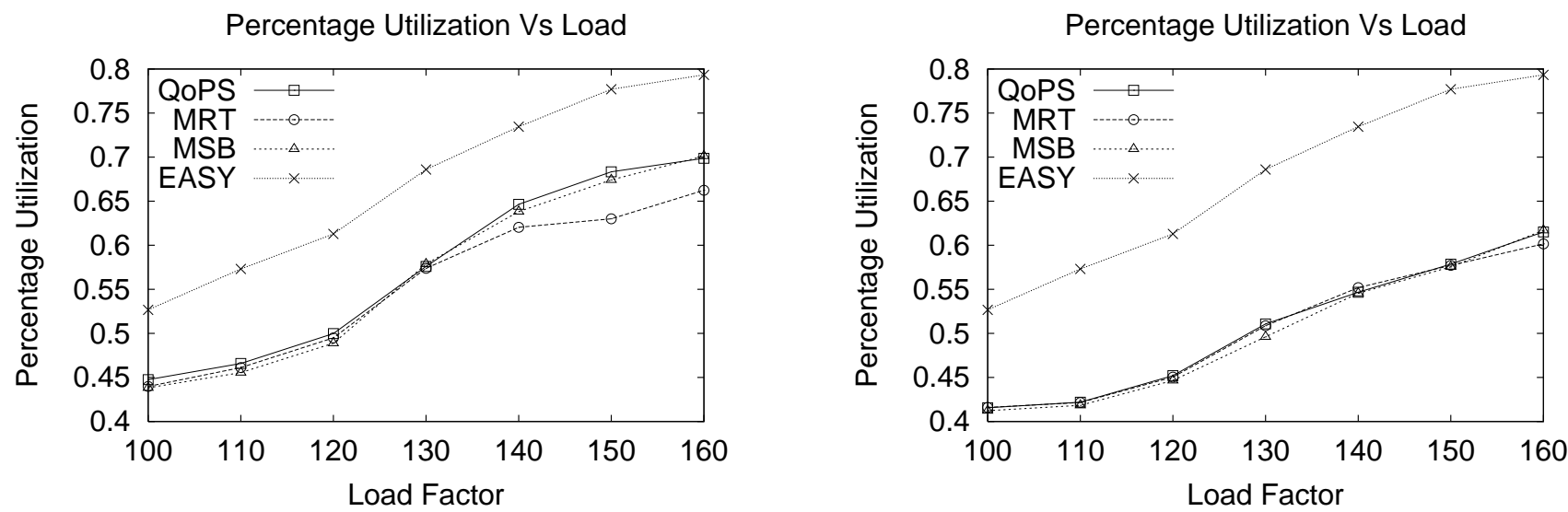

Figure 15. Utilization comparison for a mix of deadline and non-deadline jobs (Stringency Factor $=0.2$; CTC trace with job duplication): (a) Percentage of Deadline Jobs $=80 \%$, (b) Percentage of Deadline Jobs $=20 \%$

Average Response Time Vs Utilization

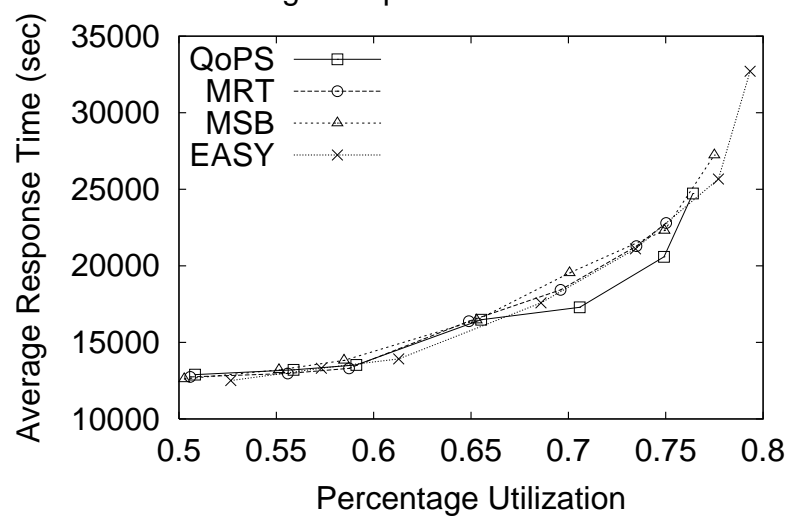

Average Slow Down Vs Utilization

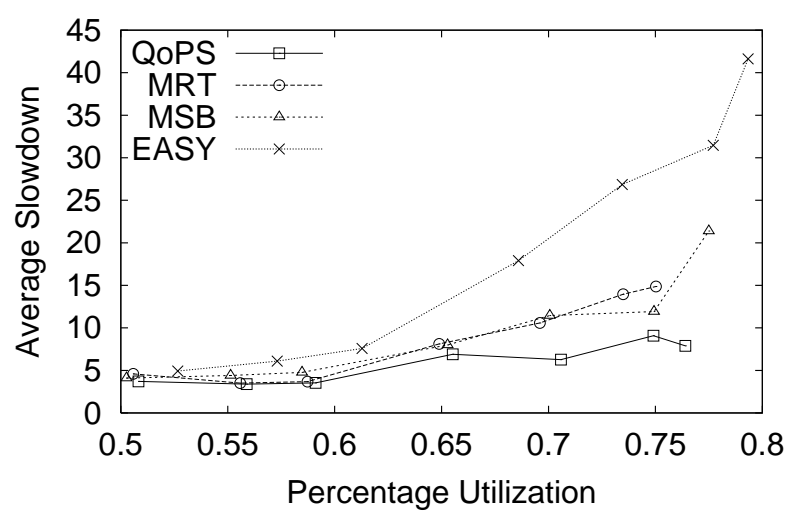

Figure 16. Performance variation of Non-deadline jobs with utilization for Percentage of Deadline Jobs $=20 \%$; Stringency Factor $=0.2$; CTC trace with job duplication (a) Variation of response time, (b) Variation of the average slowdown 

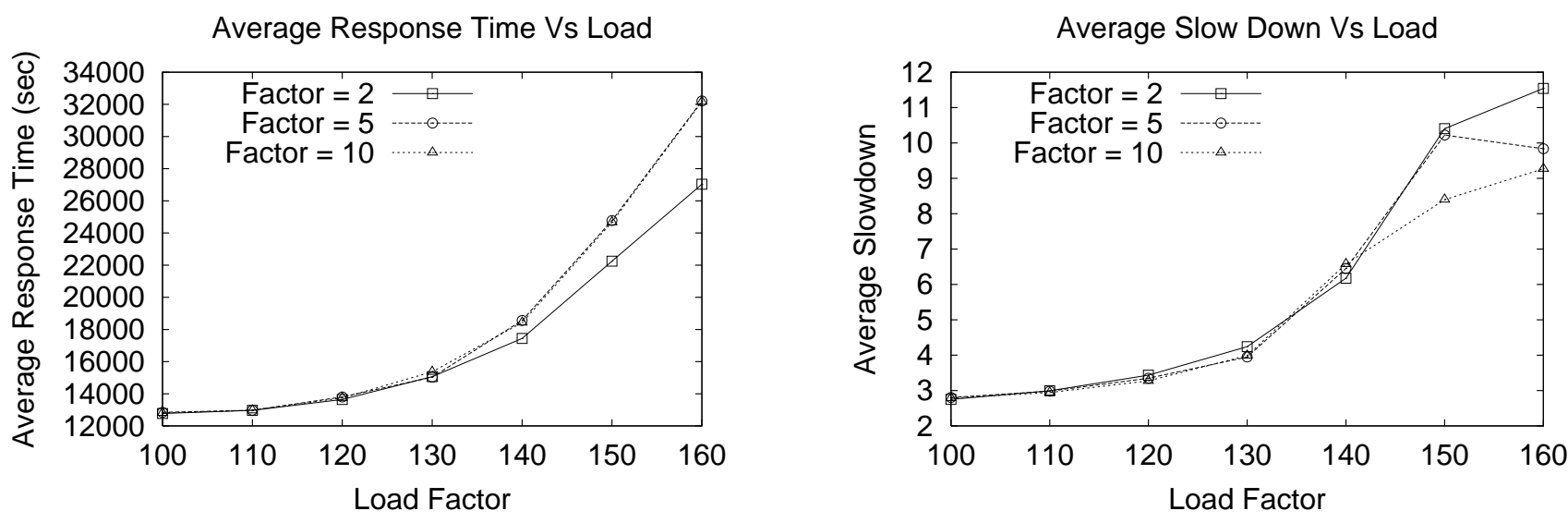

Figure 17. Performance variation of the QoPS scheme with relaxation factor for Percentage of Deadline Jobs $=80 \%$; Stringency Factor $=0.2$; CTC trace with job duplication (a) Variation of response time, (b) Variation of the average slowdown

The other issue we plan to pursue in the future is the study of the effects of inaccuracy of user estimates. The simulation studies reported in this paper assumed accurate user estimates of runtime, so that this additional factor did not complicate matters. We plan to extend our simulation studies to understand the impact of inaccurate user estimates on the performance of the QoPS scheme.

Section*Acknowledgements: We thank the anonymous referees for their valuable feedback.

\section{References}

[1] NERSC. http://hpcf.nersc.gov/accounts/priority_charging. html.

[2] Su-Hui Chiang and Mary K Vernon. Production Job Scheduling for Parallel Shared Memory Systems. In the Proceedings of the IEEE International Parallel and Distributed Processing Symposium, April 2001.

[3] Walfredo Cirne and Francine Berman. Adaptive Selection of Partition Size of Supercomputer Requests. In the Proceedings of 6th workshop on Job Scheduling Strategies for Parallel Processing, April 2000.

[4] D. G. Feitelson. Logs of Real Parallel Workloads from Production Systems.

[5] Dror G Feitelson, Larry Rudolph, Uwe Schwiegelshohn, Kenneth C Sevcik, and Parkson Wong. Theory and Practice in Parallel Job Scheduling. In the Proceedings of IEEE Workshop on Job Scheduling Strategies for Parallel Processing, 1997.

[6] Praveen Holenarsipur, Vladimir Yarmolenko, Jose Duato, D K Panda, and P Sadayappan. Characterization and Enhancement of Static Mapping Heuristics for Heterogeneous Systems. In the Proceedings of the IEEE International Symposium on High Performance Computing (HiPC), December 2000.
[7] Mohammad Islam, Pavan Balaji, P. Sadayappan, and D. K. Panda. QoPS: A QoS based scheme for Parallel Job Scheduling. Technical report, The Ohio State University, Columbus, OH, April 2003.

[8] B. Jackson, Brian Haymore, Julio Facelli, and Quinn O. Snell. Improving Cluster Utilization Through Set Based Allocation Policies. In IEEE Workshop on Scheduling and Resource Management for Cluster Computi ng, September 2001.

[9] Pete Keleher, Dmitry Zotkin, and Dejan Perkovic. Attacking the Bottlenecks in Backfilling Schedulers. In Cluster Computing: The Journal of Networks, Software Tools and Applications, March 2000.

[10] A. K. Mok. Fundamental design problems of distributed systems for the hard real-time environment. $\mathrm{PhD}$ thesis, Massachussetts Institute of Technology, Cambridge, MA, May 1983.

[11] A. K. Mok. The design of real-time programming systems based on process models. In the Proceedings of IEEE Real Time Systems Symposium, December 1984.

[12] A. K. Mok and M. L. Dertouzos. Multi-Processor Scheduling in a Hard Real-Time Environment. In the Proceedings of the Seventh Texas Conference on Computing Systems, November 1978.

[13] A. W. Mualem and D. G. Feitelson. Utilization, Predictability, Workloads and User Estimated Runtime Esti mates in Scheduling the IBM SP2 with Backfilling. In in IEEE Transactions on Parallel and Distributed Systems, volume 12, June 2001.

[14] Dejan Perkovic and Peter J Keleher. Randomization, Speculation and Adaptation in Batch Schedulers. In the Proceedings of the IEEE International Conference on Supercomputing, November 2000.

[15] Krithi Ramamritham, John A. Stankovic, and Perng-Fei Shiah. Efficient Scheduling Algorithms for Real-Time Mul- 
tiprocessor Systems. In IEEE Transactions on Parallel and Distributed Systems, volume I, April 1990.

[16] D. Talby and D. G. Feitelson. Supporting Priorities and Improving Utilization of the IBM SP2 scheduler using Slack Based Backfilling. In the Proceedings of the 13th Intl. Parallel Processing Symposium, pages 513-517, April 1997. 